\title{
LSGCN: Long Short-Term Traffic Prediction with Graph Convolutional Networks
}

\author{
Rongzhou Huang $^{1}$, Chuyin Huang ${ }^{1}$, Yubao Liu ${ }^{1,2}$, Genan Dai ${ }^{1}$ and Weiyang Kong ${ }^{1}$ \\ ${ }^{1}$ Sun Yat-Sen University, Guangzhou, China \\ ${ }^{2}$ Guangdong Key Laboratory of Big Data Analysis and Processing, Guangzhou, China \\ liuyubao@mail.sysu.edu.cn, \{huangrzh6, huangchy78, daign, kongwy3\}@mail2.sysu.edu.cn
}

\begin{abstract}
Traffic prediction is a classical spatial-temporal prediction problem with many real-world applications such as intelligent route planning, dynamic traffic management, and smart location-based applications. Due to the high nonlinearity and complexity of traffic data, deep learning approaches have attracted much interest in recent years. However, few methods are satisfied with both long and short-term prediction tasks. Target at the shortcomings of existing studies, in this paper, we propose a novel deep learning framework called Long Shortterm Graph Convolutional Networks (LSGCN) to tackle both traffic prediction tasks. In our framework, we propose a new graph attention network called cosAtt, and integrate both cosAtt and graph convolution networks (GCN) into a spatial gated block. By the spatial gated block and gated linear units convolution (GLU), LSGCN can efficiently capture complex spatial-temporal features and obtain stable prediction results. Experiments with three real-world traffic datasets verify the effectiveness of LSGCN.
\end{abstract}

\section{Introduction}

Traffic prediction is a classical spatial-temporal prediction problem whose purpose is to predict the traffic conditions (e.g. vehicle speed) of several future times based on the historical traffic observations (e.g. recorded via sensors of traffic network). The problem has been found useful in many real-world applications such as intelligent route planning, dynamic traffic management, and smart location-based applications [Wu and Tan, 2016]. In general, there are two kinds of traffic prediction tasks according to the length of prediction time, namely the short-term (5 30 minutes) and long-term (30 60 minutes) [Ostring and Sirisena, 2001; Vlahogianni et al., 2005]. The traditional methods often adopt the queuing theory, computational simulation and statistics [Ahmed and Cook, 1979; Williams and Hoel, 2003]. Since these methods rely on the stationarity assumption, they

\footnotetext{
${ }^{*}$ Yubao Liu is the corresponding author.
}
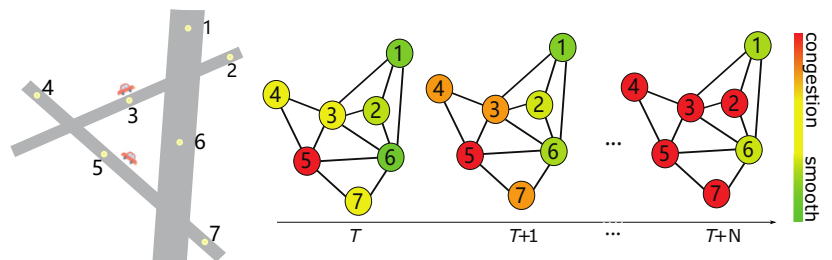

Figure 1: The left picture shows a road network with seven sensors numbered from 1 to 7 . The right pictures denote the traffic networks over time from $T$ to $T+N$. The different color of the sensors represents the degree of road congestion. For example, red means that the corresponding roads are congested and green shows the roads are smooth. If there is a sudden accident on the road with sensor 5 at time $T$, the road becomes congested. Then, at time $T+1$, the adjacent roads with sensors 3,4 and 7 may be affected by the accident and also become congested. Instead, sensors 1, 2 and 6 may be affected less since they are located at the farther roads. Moreover, since the roads with sensors 2 and 5 are similar, which are narrower than the road with sensors 1 and 6 , sensor 2 may be affected more than sensors 1 and 6 .

fail to capture complex spatial-temporal features for the prediction tasks.

Due to the high nonlinearity and complexity of traffic data, deep learning approaches have attracted much interest in recent years such as convolutional neural network $(\mathrm{CN}$ $\mathrm{N})$ and recurrent neural networks(RNN) [Wu and Tan, 2016; Ma et al., 2017; Hochreiter and Schmidhuber, 1997; Xingjian et al., 2015]. In particular, graph convolution networks $(\mathrm{GCN})$ and its variants have been used to traffic prediction tasks and often obtain promising prediction results [Yu et al., 2018; Li et al., 2018; Guo et al., 2019]. However, few methods are satisfied with both long and short-term prediction tasks. For example, STGCN [Yu et al., 2018] adopts the mechanis$\mathrm{m}$ of iterative prediction. The traffic conditions predicated in the previous iterations are used as the historical observations for the next iterations. Since the historical traffic observations may be not real values, there are errors accumulated for the prediction task. In general, the more network structure layer$\mathrm{s}$, the more errors accumulated, especially for the long-term task. On the other hand, ASTGCN [Guo et al., 2019] and DCRNN [Li et al., 2018] adopt the mechanism of non-iterative prediction, where all prediction values for multiple time steps are obtained by one unified evaluation instead of multiple iterations. So, it is hard to give considerations to both long and short-term tasks. 
Target at the shortcomings of existing studies, we propose a novel deep learning framework called Long Short-term Graph Convolutional Networks (LSGCN) to tackle both traffic prediction tasks. Intuitively, the mechanism of iterative prediction is good for the simulation of dynamic traffic conditions over time.

So, we also adopt the mechanism of iterative prediction for LSGCN. We try to enhance the performance of LSGCN from two aspects. On the one hand, we construct a simplified network structure with fewer layers and reduce the accumulated errors in each iteration. On the other hand, we design some new network layers to efficiently capture complex spatial-temporal features. Specifically, we propose a spatial gated block in which a new graph attention network called cosAtt and graph convolution networks (GCN) are integrated by the gated form. By cosAtt and GCN, the spatial gated block can precisely extract the spatial location adjacency and similar road conditions of traffic networks. For example, Figure 1 shows an application scenario can be captured by the spatial gated block. Moreover, the graph attention cosAtt enables more stable prediction results.

Our contributions are summarized as follows. (1) We propose a novel deep learning framework LSGCN for both long and short-term traffic prediction tasks. In LSGCN, we adop$\mathrm{t}$ the spatial gated block and gated linear units convolution (GLU) to capture the spatial and temporal features, respectively. We propose a new graph attention network cosAt$\mathrm{t}$ and integrate both GCN and cosAtt into the spatial gated block. (2) We evaluate our LSGCN on three real-world traffic datasets and the experimental results show that LSGCN outperforms state-of-the-art baselines.

\section{Preliminary}

\subsection{Traffic Prediction Problem}

Traffic Network. Traffic network is defined as an undirected graph $G=(V, E, W)$, where $V$ is the set of nodes, $E$ is the set of edges between two nodes, and $W \in \mathbb{R}^{N \times N}$ corresponds to the adjacency matrix of $G$. In practice, a node may represent a sensor located at the corresponding road of the traffic networks. Each node records some traffic features, such as traffic flow, vehicle speed, and road occupancy, etc.

Problem Definition. For a traffic network, let $x_{t}^{i} \in \mathbb{R}$ represents the traffic feature value of the $i^{t h}$ node at time step $t$, and $x_{t} \in \mathbb{R}^{N}$ represents the traffic feature values of all nodes at time step $t$. Given history traffic data $X=$ $\left(x_{1}, x_{2}, \ldots, x_{\tau}\right)^{T} \in \mathbb{R}^{N \times \tau}$, the purpose of traffic prediction is to predict the traffic features of all nodes in future $T_{p}$ time steps, namely $Y^{*}=\left(y_{1}, y_{2}, \ldots, y_{T_{p}}\right)^{T} \in \mathbb{R}^{N \times T_{p}}$. Without loss of generality, we consider the feature of vehicle speed in the following.

\subsection{Attention on Graphs}

The input to graph attention networks (GAT) is a set of node features, $\mathcal{Q}=\left\{\overrightarrow{q_{1}}, \overrightarrow{q_{2}}, \ldots, \overrightarrow{q_{N}}\right\}$, where $\overrightarrow{q_{i}^{\prime}} \in \mathbb{R}^{F}$ and $N$ is the number of nodes, and $F$ is the number of features in each node. The output is also a new set of node features (of potentially different cardinality $\left.F^{\prime}\right), \mathcal{Q}^{\prime}=\left\{\overrightarrow{q_{1}^{\prime}}, \overrightarrow{q_{2}^{\prime}}, \ldots, \overrightarrow{q_{N}^{\prime}}\right\}$, where $\overrightarrow{q_{i}^{\prime}} \in \mathbb{R}^{F^{\prime}}$. The attention coefficient for any pair of $\overrightarrow{q_{i}}$ and $\overrightarrow{q_{j}}$ is given as follows.

$$
e_{i j}=A\left(\overrightarrow{q_{i}} w_{i}, \overrightarrow{q_{j}} w_{j}\right), w_{i}, w_{j} \in F \times F^{\prime}
$$

where $w_{i}$ and $w_{j}$ are weight matrix components, and $A(\cdot)$ is the attention mechanism operation function. Then, we normalize the attention coefficient $e_{i j}$ among node neighborhoods and achieve the output as follows.

$$
\begin{gathered}
a_{i j}=\operatorname{softmax}\left(e_{i j}\right)=\frac{\exp \left(e_{i j}\right)}{\sum_{k \in \mathcal{N}_{i}} \exp \left(e_{i k}\right)} \\
\left.\overrightarrow{q_{i}^{\prime}}=\sigma\left(\sum_{j \in \mathcal{N}_{i}} a_{i j} \overrightarrow{q_{j}} w_{j}\right)\right)
\end{gathered}
$$

where $\mathcal{N}_{i}$ is the set of neighbours of node $i$ in the graph and $\sigma$ is an activation function [Velickovic et al., 2017].

\section{Proposed Model}

\subsection{Network Architecture}

LSGCN is composed of five layers, namely two gated linear units convolutional layers (GLUs), spatial gated block, convolution-unified layer and fully connection layer. The spatial gated block is between two GLUs as shown in Figure 2. Our network structure has fewer layers compared with STGCN [Yu et al., 2018]. LSGCN adopts the mechanism of iterative prediction as follows. In each iteration, we first obtain $H^{\prime}=\left(h_{1}^{\prime}, h_{2}^{\prime}, \ldots, h_{\tau-K_{T}+1}^{\prime}\right)^{T} \in \mathbb{R}^{N \times\left(\tau-K_{T}+1\right) \times C_{1}}$ by the first GLU layer, where $K_{T}$ is the width of $1 \mathrm{D}$ convolution kernel and $C_{1}$ is the channel numbers. Then, we can obtain $H^{\prime \prime}=\left(h_{1}^{\prime \prime}, h_{2}^{\prime \prime}, \ldots, h_{\tau-K_{T}+1}^{\prime \prime}\right)^{T} \in \mathbb{R}^{N \times\left(\tau-K_{T}+1\right) \times C_{1}}$ by spatial gated block, which is based on $H^{\prime}$ and the adjacency matrix $W$. Next, $H^{\prime \prime}$ is processed by the second GLU, and we can obtain $H^{\prime \prime \prime}=\left(h_{1}^{\prime \prime \prime}, h_{2}^{\prime \prime \prime}, \ldots, h_{\tau-2 \times\left(K_{T}-1\right)}^{\prime \prime \prime}\right)^{T} \in$ $\mathbb{R}^{N \times\left(\tau-2 \times\left(K_{T}-1\right)\right) \times C_{2}}$, where $C_{2}$ is the second channel numbers. Finally, the output is achieved by the convolution unified layer and fully connection layer, namely $Y=\left(y_{1}\right)^{T} \in$ $\mathbb{R}^{N \times 1 \times 1}$. Note that the obtained $Y$ is added into the input for next iteration. For example, the input for the second iteration is $\left\{x_{2}, \ldots, x_{i}, \ldots, x_{\tau}, y_{1}\right\}$. The iteration process is halted after $T_{p}$ iterations. The adjacent road conditions are important in short-term task, the similar road conditions more so in longterm task. They can be captured by GCN and cosAtt of the network model, respectively.

\subsection{Spatial Gated Block for Extracting Spatial Features}

We consider two main spatial features, namely spatial location adjacency and road condition similarity. We use GCN to extract the spatial location adjacency and a new graph attention network cosAtt to extract road condition similarity.

Inspired by the sequential gate structure, such as LSTM [Hochreiter and Schmidhuber, 1997] and GRU [Chung et $a l ., 2014]$, we adopt the gate structure to integrate both components. We use the sigmoid function to map the results of GCN to the interval $(0,1)$ and control the output of cosAtt. Intuitively, GCN determines how much information generated by cosAtt can flow into the next layer based on the 


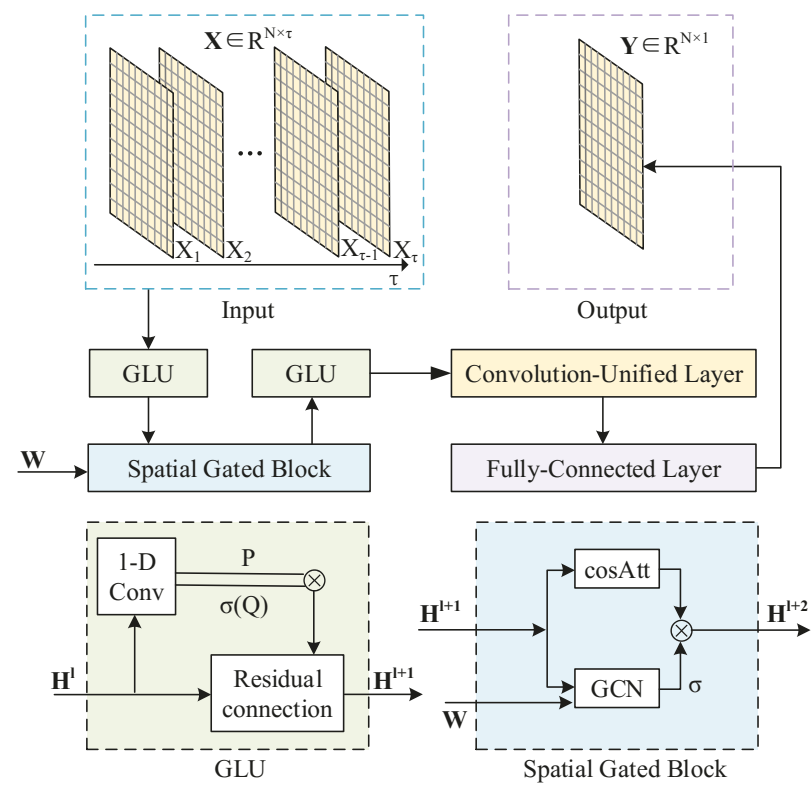

Figure 2: Network architecture of LSGCN. $P$ and $Q$ are the output$\mathrm{s}$ of 1-D Conv, $\sigma$ represents the sigmoid activation function and $\otimes$ represents the element-wise Hadamard product between two branches.

topological structure (i.e., spatial location adjacency) of traffic networks.

As shown in Figure 2, the spatial gated block works as follows. First, we pass the $H^{l+1}$ generated by the first GLU and the adjacency matrix $W$ into GCN. The results of GCN then go through the sigmoid activation function. On the other hand, we make a copy of $H^{l+1}$ and pass it to cosAtt. Finally, $H^{l+2}$ is achieved by the element-wise Hadamard product on the results generated before.

Graph Attention cosAtt. Based on the observation that the roads adjacent to each other in a traffic network often have similar road conditions as shown in Figure 1. We design a new graph attention cosAtt to extract the similar road conditions of traffic networks. In cosAtt, we adopt global graph attention networks to learn the similar conditions of any two roads of traffic network instead of neighbours in graph attention networks (GAT).

Initially, node $H=\left\{h_{1}, \ldots, h_{i}, \ldots, h_{N}\right\}, h_{i} \in \mathbb{R}^{N \times T \times C_{i}}$ is passed into cosAtt, where $T$ represents time steps and $C_{i}$ is input channels. We define cosAtt as follows.

$$
\begin{gathered}
e_{i j}=\cos \left(h_{i}, h_{j}\right) \cdot w_{i j}=\frac{h_{i} \times h_{j}^{T} \cdot w_{i j}}{\left\|h_{i}\right\| \times\left\|h_{j}\right\|} \\
a_{i j}=\operatorname{sigmoid}\left(e_{i j}\right) \\
{\cos A t t_{i}}=\sum_{j \in \widetilde{N}_{i}} a_{i j} h_{j} w_{i j}
\end{gathered}
$$

where $\widetilde{\mathcal{N}}_{i}$ is all nodes in graph except node $i$.

Next, we show the key differences between cosAtt and traditional GAT. First, the weight distribution of cosAtt is used as the output, whereas the weight distribution of GAT is added into the original features. On the other hand, for any given two features $h_{i}$ and $h_{j}$, the similarity value between them is constant (i.e., $\cos \left(h_{i}, h_{j}\right)$ ). The value of $e_{i j}$ is relatively stable and only adjusted by the weight matrix $W$ (i.e., $w_{i j}$ ). In contrary to cosAtt, the value of $e_{i j}$ in GAT may be unstable since we have to learn them by the attention function and the weight matrix $W$ as shown in Eq.(1).

Note that we use the sigmoid instead of the softmax activation function in our model. This is because that the softmax activation function often results in denominator underflow and program crash since the number of neighbors (i.e., all nodes except the target node itself) for each node may be huge.

The details on GCN are as follows. Based on the conception of spectral graph convolution, the graph convolution operator " $* \mathcal{G}$ " can be represented as the multiplication of a signal $x \in \mathbb{R}^{N}$ with a kernel $\Theta$.

$$
\Theta *_{\mathcal{G}} x=\Theta(L) x=\Theta\left(U \Lambda U^{T}\right) x=U \Theta(\Lambda) U^{T} x
$$

In the equation, graph Fourier basis $U \in \mathbb{R}^{N \times N}$ corresponds to the matrix of eigenvectors of the normalized graph Laplacian $L=I_{N}-D^{-\frac{1}{2}} W D^{-\frac{1}{2}}=U \Lambda U^{T} \in \mathbb{R}^{N \times N}$, where $I_{N}$ is an identity matrix and $D \in \mathbb{R}^{N \times N}$ is the diagonal degree matrix with $D_{i i}=\sum_{j} W_{i j} . \Lambda \in \mathbb{R}^{N \times N}$ corresponds to the diagonal matrix of eigenvalues of $L$ and filter $\Theta(\Lambda)$ is also a diagonal matrix [Shuman et al., 2013].

Since the computation of Eq.(7) is expensive, we adopt the Chebyshev polynomials approximation in our implementation to speed up the computation of GCN.

$$
\Theta *_{\mathcal{G}} x=\Theta(L) x \approx \sum_{k=0}^{K-1} \theta_{k} T_{k}(\tilde{L}) x
$$

where $\theta \in \mathbb{R}^{K}$ is a vector of polynomial coefficients, $K$ is the kernel size of graph convolution, and $T_{k}(\tilde{L}) \in \mathbb{R}^{N \times N}$ corresponds to the Chebyshev polynomial of order $k$ evaluated at the scaled Laplacian $\tilde{L}=2 L / \lambda_{\max }-I_{N}$. The computation of Eq. (8) takes $\mathcal{O}(K \cdot|E|)$ time [Defferrard et al., 2016].

\subsection{Gated Linear Units Convolution Layer for Extracting Temporal Features}

We use GLU to capture dynamic behaviors of temporal features, which is similar to STGCN [Yu et al., 2018]. As shown in Figure 2, there are two GLUs each of which consists of a $1 \mathrm{D}$ convolution with a width- $K_{T}$ kernel and a residual connection.

In general, GLU works as follows. The input is $X=$ $\left\{x_{1}, x_{2}, \ldots, x_{i}, \ldots, x_{N}\right\}, x_{i} \in \mathbb{R}^{N \times \tau \times C_{i}}\left(C_{i}=1\right.$ for the first GLU). Next, we use convolutional kernels $\Theta \in$ $\mathbb{R}^{K_{t} \times C_{i} \times 2 C_{o}}$ to obtain the convolutional result $Z=[P Q] \in$ $\mathbb{R}^{N \times\left(\tau-K_{T}+1\right) \times 2 C_{o}}$, where $C_{o}$ is the size of the feature set generated by GLU. Note that the number of $1 \mathrm{D}$ convolutional kernels is set as $2 \cdot C_{o}$. The output through $1 \mathrm{D}$ convolutional layer is divided into two equal parts, namely $P$ and $Q$. $P$ is the output of the first half convolution kernels and $Q$ is of the second half convolution kernels. Then, the element-wise Hadamard product of $P$ and $\sigma(Q)$ is computed and added into the input of the residual connection. If the number of input 


\begin{tabular}{c|c|c|c}
\hline & PeMSD4 & PeMSD7 & PeMSD8 \\
\hline \#Nodes & 307 & 228 & 170 \\
\hline \#Edges & 340 & 832 & 295 \\
\hline \#Time steps & 16992 & 12672 & 17856 \\
\hline \multirow{2}{*}{ Time span } & $2018 / 1-$ & $2012 / 5-2012 / 6$ & $2016 / 7-$ \\
& $2018 / 2$ & (weekdays) & $2016 / 8$ \\
\hline Time interval & \multicolumn{3}{|c}{ 5mins } \\
\hline Daily range & \multicolumn{3}{|c}{$0: 00-24: 00$} \\
\hline
\end{tabular}

Table 1: The details for the datasets

dimension is less than that of the output dimension, the input dimension is filled with zero, and vice versa. Finally, we can reduce the time steps to $\tau-2 \times\left(K_{T}-1\right)(>0)$ based on two GLUs.

\subsection{Convolution Unified Layer}

In the mechanism of iterative prediction, we need to output single prediction value for each time step in each iteration process. So, it is necessary to reduce dimension on time axis. As shown Figure 2, LSGCN adopts the convolution unified layer to merge the $\tau-2 \times\left(K_{T}-1\right)$ time steps into single time step with casual convolution. In general, the sequence with $\tau-2 \times\left(K_{T}-1\right)$ time steps is a relatively long sequence. For a long sequence, the casual convolution fuse time steps in proportion, so we can obtain a reasonable time step as the output of this layer.

\subsection{Loss Function}

We use L2 loss to measure the performance of our model which is defined as follows,

$$
L\left(\hat{y} ; W_{\theta}\right)=\sum_{t}\left\|\hat{y}\left(x_{t-\tau}, \ldots, x_{t}, W_{\theta}\right)-x_{t+1}\right\|^{2}
$$

where $\hat{y}(\cdot)$ is the prediction value, $x_{t+1}$ denotes the ground truth and $W_{\theta}$ represents all trainable parameters.

\section{Experiment}

\subsection{Dataset Description}

In the experiment, we use three real-world traffic datasets, namely PeMSD4, PeMSD7 and PeMSD8, which are collected by California Performance of Transportation(PeMS) [Chen et al., 2001] and widely used in the previous studies such as STGCN and ASTGCN [Yu et al., 2018; Guo et al., 2019]. More details for the datasets are in Table 1. The PeMS sensor network is shown in Figure 3.

PeMSD4. It refers to the traffic data in San Francisco Bay Area, with 307 sensors on 29 roads. The dataset spanned from January to February in 2018. The first 47 days are used as training set, and the remaining as validation and test set.

PeMSD7. It refers to the traffic data in District 7 of California, with 228 sensors and the time range is in the weekdays from May to June in 2012. The first 34 days are used as training set, and the remaining as validation and test set.

PeMSD8. It refers to the traffic data in San Bernardino from July to August in 2016, with 170 detectors on 8 roads. The first 50 days are used as training set, and the remaining as validation and test set.
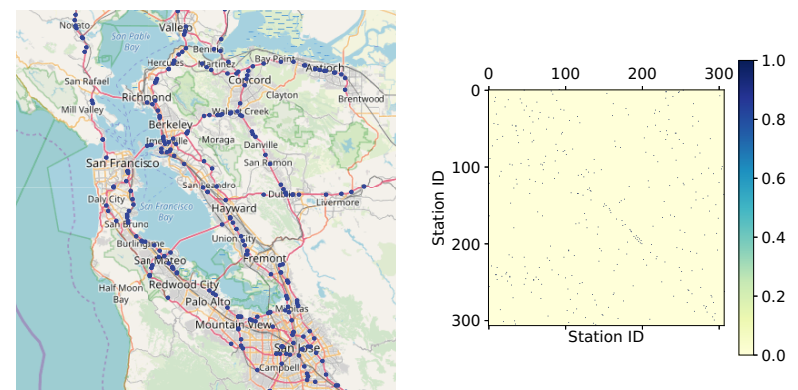

Figure 3: PeMS sensor network in District 4 of California (left). Sensor stations are denoted by dots. Heat map of weighted adjacency matrix in PeMSD4 (right).

\subsection{Data Preprocessing}

We set the standard time interval as 5 minutes. Therefore, every sensor contains 288 traffic data points per day. We use linear interpolation method to fill the missing values and ZScore method to normalize the input data.

For PeMSD4 and PeMSD8, the weighted adjacency matrix $W$ can be formed as,

$$
w_{i j}=\left\{\begin{array}{l}
\exp \left(-\frac{d_{i j}^{2}}{\delta}\right), \text { if } i, j \text { are neighbours } \\
0, \text { if } i, j \text { aren't neighbours }
\end{array}\right.
$$

where $\delta$ is the threshold to control the distribution of $W$ and is set to be $0.1, w_{i j}$ is the edge weight which is related to $d_{i j}$ (the distance between station $i$ and $j$ ).

For PeMSD7, the weighted adjacency matrix $W$ can be formed as,

$$
w_{i j}=\left\{\begin{array}{l}
\exp \left(-\frac{d_{i j}^{2}}{\delta}\right), i \neq j \text { and } \exp \left(-\frac{d_{i j}^{2}}{\delta}\right) \geq \epsilon \\
0, \text { otherwise }
\end{array}\right.
$$

where $\delta$ and $\epsilon$ are the thresholds to control the distribution and sparsity of $W$ and are set to be $\delta=0.1$ and $\epsilon=0.5$, respectively. $w_{i j}$ is the edge weight which is related to $d_{i j}$ (the distance between station $i$ and $j$ ).

\subsection{Experimental Settings}

All experiments are performed on a Linux server (CPU: Intel(R) Xeon(R) CPU E5-2620 v4 @ 2.10GHz, GPU: GeForce RTX $2080 \mathrm{Ti}$ ). The grid search strategy is executed to locate the best parameters on validations. All the tests adopt 60 minutes as the history time window, a.k.a. 12 observed data points $(\tau=12)$ are used to forecast traffic conditions in the next 15, 30, 45 and 60 minutes $\left(T_{p}=3,6,9,12\right)$.

Evaluation Metric \& Baselines. We adopt Mean Absolute Errors (MAE), Mean Absolute Percentage Errors (MAPE), and Root Mean Squared Errors (RMSE) to measure the performance of different methods. The baselines are as follows.

- HA: Historical Average. Here, we use the average value of the last 12 times slices to predict the next value.

- ARIMA [Williams and Hoel, 2003]: Auto-Regressive Integrated Moving Average method, which is widely used in time series prediction. 


\begin{tabular}{|c|c|c|c|}
\hline \multirow{2}{*}{ Model } & \multicolumn{3}{|c|}{ PeMSD4 (15/ 30/ 45/ 60 min) } \\
\hline & MAE & MAPE (\%) & RMSE \\
\hline HA & 2.54 & 5.56 & 4.96 \\
\hline ARIMA & $2.51 / 2.75 / 2.96 / 3.21$ & $5.32 / 5.69 / 6.01 / 6.56$ & $5.72 / 6.34 / 6.85 / 7.36$ \\
\hline DCRNN & $1.35 / 1.77 / 2.04 / 2.26$ & 2.68/3.71/ $4.48 / 5.10$ & $2.94 / 4.06 / 4.77 / 5.28$ \\
\hline STGCN & $1.47 / 1.93 / 2.26 / 2.55$ & $2.92 / 3.98 / \overline{4.73 / 5.39}$ & $3.01 / 4.21 / 5.01 / 5.65$ \\
\hline ASTGCN & $2.12 / 2.42 / 2.60 / 2.73$ & $4.16 / 4.80 / 5.20 / 5.46$ & $3.96 / 4.59 / 4.97 / 5.21$ \\
\hline LSGCN & $\underline{1.45 / \underline{1.82 / 2.04 / 2.22}}$ & $\underline{2.90 / \underline{3.84} / 4.42 / 4.85}$ & $2.93 / 3.92 / 4.47 / \overline{4.83}$ \\
\hline \multirow{2}{*}{ Model } & \multicolumn{3}{|c|}{ PeMSD7 (15/30/45/ 60 min) } \\
\hline & MAE & MAPE (\%) & RMSE \\
\hline HA & 4.01 & 10.61 & 7.20 \\
\hline ARIMA & $5.57 / 5.94 / 6.27 / 6.68$ & $13.04 / 14.01 / 15.01 / 16.78$ & $9.00 / 9.22 / 9.43 / 9.68$ \\
\hline DCRNN & $2.22 / 3.04 / 3.64 / 4.15$ & $5.16 / 7.46 / 9.26 / 10.82$ & $4.25 / 6.02 / 7.24 / 8.20$ \\
\hline STGCN & $\overline{2.24} / \overline{3.04} / \underline{3.61 / 4.08}$ & $5.28 / \overline{7.46} / \underline{9.00 / 10.23}$ & $\underline{4.01 / ~} \underline{5.74 / 6.85 / 7.69}$ \\
\hline ASTGCN & $2.85 / 3.35 / \overline{3.70} / 3.96$ & $7.25 / 8.67 / \overline{9.73} / \overline{10.53}$ & $\overline{5.15} / \overline{6.12} / 6.77 / 7.20$ \\
\hline LSGCN & $2.22 / 2.96 / 3.43 / \overline{3.81}$ & $5.14 / 7.18 / 8.51 / 9.60$ & $3.98 / 5.47 / \overline{6.39} / \overline{7.09}$ \\
\hline \multirow{2}{*}{ Model } & \multicolumn{3}{|c|}{ PeMSD8 (15/ 30/ 45/ 60 min) } \\
\hline & MAE & MAPE (\%) & RMSE \\
\hline HA & 1.98 & 3.94 & 4.11 \\
\hline ARIMA & $1.90 / 2.12 / 2.43 / 2.79$ & $5.11 / 5.21 / 5.46 / 5.62$ & $4.87 / 5.24 / 5.63 / 6.22$ \\
\hline DCRNN & $1.17 / 1.49 / 1.71 / 1.87$ & $2.32 / 3.21 / 3.83 / 4.28$ & $\underline{2.59 / 3.56 / 4.13 / 4.50}$ \\
\hline STGCN & $\overline{1.19} / \overline{1.59} / \overline{1.92} / \overline{2.25}$ & $\overline{2.34} / \overline{3.24} / \overline{3.91} / 4.54$ & $\overline{2.62} / \overline{3.61} / 4.21 / 4.68$ \\
\hline ASTGCN & $1.49 / 1.67 / 1.81 / 1.89$ & $3.16 / 3.59 / 3.98 / 4.22$ & $3.18 / 3.69 / 3.92 / 4.13$ \\
\hline LSGCN & $1.16 / 1.46 / 1.66 / 1.81$ & $2.24 / 3.02 / 3.51 / \overline{3.89}$ & $2.45 / 3.28 / \overline{3.75} / \overline{4.11}$ \\
\hline
\end{tabular}

Table 2: Performance comparison of different approaches on the datasets PeMSD4, PeMSD7 and PeMSD8.

- DCRNN [Li et al., 2018]: Diffusion Convolution Recurrent Neural Network, which combines graph convolution with recurrent neural networks in an encoderdecoder manner.

- STGCN [Yu et al., 2018]: Spatial-Temporal Graph Convolutional Network, which combines graph convolution with gated temporal convolution.

- ASTGCN [Guo et al., 2019]: Attention based SpatialTemporal Graph Convolution Network, which combines the spatial-temporal attention mechanism and the spatial-temporal convolution.

LSGCN Model. The batch size of PeMSD4, PeMSD7 and PeMSD8 are 32, 32 and 16, respectively. The hyperparameters are set as follows. For all datasets, the channels of the first GLU, GCN, cosAtt, the second GLU are 32, 32, 32 and 64, respectively. We set both the graph convolution kernel size $K$ and GLU convolution kernel size $K_{t}$ to 3 . Our model is trained by minimizing the mean square error with RMSprop optimizer for 60 epochs. The initial learning rate is set as $10^{-3}$ with a decay rate of 0.7 after every 5 epochs.

\subsection{Experiment Results}

As shown in Table 2, LSGCN performs well in both longterm and short-term prediction for three evaluation metrics. In detail, in the three datasets, it performs very well, especially in the long-term prediction of PeMSD4, both long-term prediction and short-term prediction of PeMSD7 and PeMSD8. In the short-term prediction of PeMSD4, it also achieves the second best. The results show that LSGCN can efficiently capture the spatial-temporal features for the prediction tasks.
In particular, STGCN is also based on the mechanism of iterative prediction, but it causes more accumulation errors than LSGCN since it consists of more network structure layers.

We can easily observe that the traditional statistical methods (i.e., HA and ARIMA) often have poor performance since they cannot efficiently handle the complex spatial-temporal data.

In general, since there are lots of accumulated errors in STGCN for the prediction tasks, ASTGCN and DCRNN have better performance than STGCN. In detail, STGCN is not better than DCRNN in the short-term tasks and not better than ASTGCN in the long-term tasks. DCRNN adopts diffusion convolution which is beneficial to capture complex spatial topology information. Besides, it can accurately calculate the probability from the target sensor to neighbour sensors by using a finite step random walk to express the diffusion process. Thus, DCRNN has the best performance in the metrics of MAE and MAPE for the complex traffic networks of PeMSD4. But, DCRNN also adopts GRU structure for time series which often causes information loss in the long-term tasks.

ASTGCN considers the periodicity in prediction tasks and various traffic characteristics related to the tasks. So, it performs well in the long-term prediction of PeMSD4 and PeMSD8. But, ASTGCN is not better than STGCN in the long-term prediction of PeMSD7. This is because that the neighbor information is beneficial to the prediction tasks, which is removed by the threshold from PeMSD7, and saved in PeMSD4 and PeMSD8.

Test in extreme case. In particular, we test our LSGCN in some extreme cases, namely morning peak and evening rush 


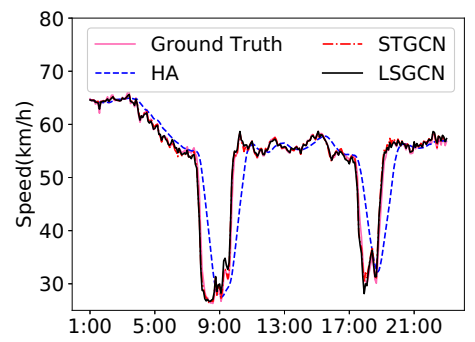

Figure 4: Speed prediction in the morning peak and evening rush hours of the dataset PeMSD4.
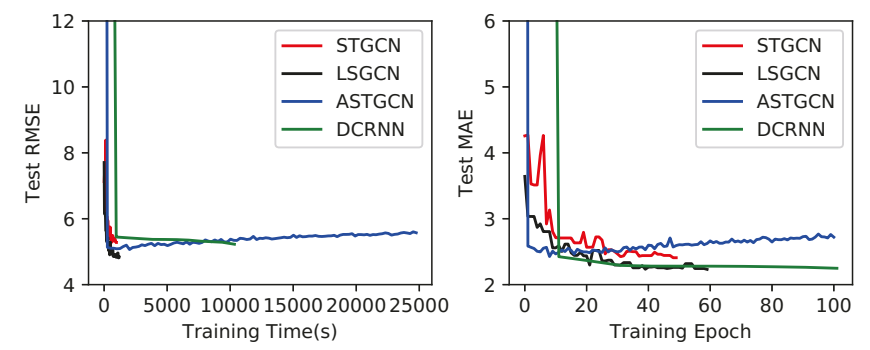

Figure 5: Test RMSE versus the training time(left); Test MAE versus the number of training epochs(right).(PeMSD4)

hours. As shown in Figure 4, Due to the efficient spatial gated block, LSGCN is capable of fast response to the dynamic changes in extreme cases. The trend of rush hours captured by LSGCN is more accurate than the other methods. Besides, the ending of the rush hours detected by LSGCN is earlier than the others.

Training Efficiency and Generalization. We plot the RMSE and MAE of the test set of PeMSD4 during the training process to further investigate the performance of different deep learning models. As shown in Figure 5, it is obvious that our LSGCN can achieve faster training and easier convergence than DCRNN and ASTGCN. Specifically, LSGCN and STGCN need almost the same training time, about 1,000 seconds, whereas DCRNN and ASTGCN need about 10,000 and 25,000 seconds, respectively. This is because that more parameters are used for the training process of DCRNN and ASTGCN.

Effect of cosAtt. By replacing the proposed cosAtt of LSGCN with traditional graph attention, we can obtain a modified version called LSGCN(GAT). We compare LSGCN and LSGCN(GAT) to test the changes in prediction results. For each prediction task, both methods are executed 10 times with the same hyperparameters. Then, the maximum and minimum values among all evaluation results for each metric are reported, respectively. As shown in Table 3, the metric value changes for LSGCN are often smaller than LSGCN(GAT), so cosAtt enables more stable prediction results.

\section{Related Work}

Deep learning models. Some solutions for traffic prediction are proposed based on deep learning models. Zhang et al. [2016] convert the road network to a regular 2-D grid and

\begin{tabular}{ccccc}
\hline & min & MAPE $(\%)$ & MAE & RMSE \\
\hline & 15 & $2.37 \pm 0.06$ & $1.19 \pm 0.02$ & $2.62 \pm 0.08$ \\
LSGCN & 30 & $3.11 \pm 0.08$ & $1.49 \pm 0.02$ & $3.46 \pm 0.07$ \\
(GAT) & 45 & $3.55 \pm \mathbf{0 . 0 9}$ & $1.68 \pm \mathbf{0 . 0 3}$ & $3.88 \pm 0.08$ \\
& 60 & $3.87 \pm 0.12$ & $1.82 \pm 0.06$ & $4.16 \pm 0.10$ \\
\hline & 15 & $2.23 \pm \mathbf{0 . 0 4}$ & $1.17 \pm \mathbf{0 . 0 1}$ & $2.47 \pm \mathbf{0 . 0 3}$ \\
LSGCN & 30 & $2.96 \pm \mathbf{0 . 0 8}$ & $1.48 \pm \mathbf{0 . 0 2}$ & $3.28 \pm \mathbf{0 . 0 5}$ \\
& 45 & $3.43 \pm 0.10$ & $1.69 \pm 0.03$ & $3.77 \pm \mathbf{0 . 0 7}$ \\
& 60 & $3.80 \pm \mathbf{0 . 1 1}$ & $1.85 \pm \mathbf{0 . 0 5}$ & $4.14 \pm \mathbf{0 . 0 8}$ \\
\hline
\end{tabular}

Table 3: Prediction value changes on PeMSD8

apply CNN to capture adjacent relations among the traffic network. Yao et al. [2018] proposed a method to predict traffic by integrating CNN and long-short term memory (LSTM) to jointly model both spatial and temporal dependencies. Different from these methods, both GCN and GAT are integrated in our proposed model.

Graph convolutional networks(GCN). Bruna et al. [2013] propose GCN based on the spectral graph theory. Defferard et al. [2016] and Kipf et al. [2016] propose some improve methods for the computation of GCN. Based on GCN, Yu et al. [2018] propose STGCN to tackle the time series prediction problem in the traffic domain. $\mathrm{Wu}$ et al.[2019] propose Graph Wavenet to precisely capture the hidden spatial dependency in the data. In addition to GCN, our model contains an additional GAT which is different from these methods.

Attention mechanism. It has been widely utilized in various domains such as natural language processing, speech recognition and image caption [Vaswani et al., 2017; Shen et al., 2018]. Recently, researchers apply attention mechanism to graph [Velickovic et al., 2017; Liu et al., 2018] and traffic prediction. Liang et al. [2018] propose a novel multi-level attention-based network. Guo et al. [2019] propose ASTGCN and Zheng et al. [2019] propose GMAN. Different from these methods, our graph attention is based on the road condition similarity.

\section{Conclusion}

In this paper, we propose a new graph convolutional networks model LSGCN for long and short-term traffic prediction. In LSGCN, we integrate both a new graph attention network $\cos A t t$ and GCN to precisely capture the spatial features and at the same adopt the GLU to capture the temporal features. The experiments on real traffic networks verify the effectiveness of LSGCN. In the future, we will consider our proposed model for more general spatio-temporal structured sequence forecasting such as preference prediction in recommendation systems.

\section{Acknowledgements}

This paper is supported by the National Nature Science Foundation of China (61572537, U1501252). 


\section{References}

[Ahmed and Cook, 1979] Mohammed S Ahmed and Allen R Cook. Analysis of freeway traffic time-series data by using Box-Jenkins techniques. Number 722. 1979.

[Bruna et al., 2013] Joan Bruna, Wojciech Zaremba, Arthur Szlam, and Yann LeCun. Spectral networks and locally connected networks on graphs. arXiv preprint arXiv:1312.6203, 2013.

[Chen et al., 2001] Chao Chen, Karl Petty, Alexander Skabardonis, Pravin Varaiya, and Zhanfeng Jia. Freeway performance measurement system: mining loop detector data. Transportation Research Record, 1748(1):96-102, 2001.

[Chung et al., 2014] Junyoung Chung, Caglar Gulcehre, Kyunghyun Cho, and Yoshua Bengio. Empirical evaluation of gated recurrent neural networks on sequence modeling. arXiv: Neural and Evolutionary Computing, 2014.

[Defferrard et al., 2016] Michaël Defferrard, Xavier Bresson, and Pierre Vandergheynst. Convolutional neural networks on graphs with fast localized spectral filtering. In NIPS, pages 3844-3852, 2016.

[Guo et al., 2019] Shengnan Guo, Youfang Lin, Ning Feng, Chao Song, and Huaiyu Wan. Attention based spatialtemporal graph convolutional networks for traffic flow forecasting. In $A A A I$, volume 33, pages 922-929, 2019.

[Hochreiter and Schmidhuber, 1997] Sepp Hochreiter and Jürgen Schmidhuber. Long short-term memory. Neural computation, 9(8):1735-1780, 1997.

[Kipf and Welling, 2016] Thomas N Kipf and Max Welling. Semi-supervised classification with graph convolutional networks. arXiv preprint arXiv:1609.02907, 2016.

[Li et al., 2018] Yaguang Li, Rose Yu, Cyrus Shahabi, and Yan Liu. Diffusion convolutional recurrent neural network: Data-driven traffic forecasting. In ICLR, 2018.

[Liang et al., 2018] Yuxuan Liang, Songyu Ke, Junbo Zhang, Xiuwen Yi, and Yu Zheng. Geoman: Multi-level attention networks for geo-sensory time series prediction. In IJCAI, pages 3428-3434, 2018.

[Liu et al., 2018] Lingbo Liu, Ruimao Zhang, Jiefeng Peng, Guanbin Li, Bowen Du, and Liang Lin. Attentive crowd flow machines. pages 1553-1561, 2018.

[Ma et al., 2017] Xiaolei Ma, Zhuang Dai, Zhengbing He, Jihui Ma, Yong Wang, and Yunpeng Wang. Learning traffic as images: a deep convolutional neural network for large-scale transportation network speed prediction. Sensors, 17(4):818, 2017.

[Ostring and Sirisena, 2001] Sven AM Ostring and Harsha Sirisena. The influence of long-range dependence on traffic prediction. In ICC 2001. IEEE International Conference on Communications. Conference Record (Cat. No. 01CH37240), volume 4, pages 1000-1005. IEEE, 2001.

[Shen et al., 2018] Tao Shen, Tianyi Zhou, Guodong Long, Jing Jiang, Shirui Pan, and Chengqi Zhang. Disan: Directional self-attention network for $\mathrm{rnn} / \mathrm{cnn}$-free language understanding. In $A A A I, 2018$.
[Shuman et al., 2013] David I Shuman, Sunil K Narang, Pascal Frossard, Antonio Ortega, and Pierre Vandergheynst. The emerging field of signal processing on graphs: Extending high-dimensional data analysis to networks and other irregular domains. IEEE signal processing magazine, 30(3):83-98, 2013.

[Vaswani et al., 2017] Ashish Vaswani, Noam Shazeer, Niki Parmar, Jakob Uszkoreit, Llion Jones, Aidan N Gomez, Łukasz Kaiser, and Illia Polosukhin. Attention is all you need. In NIPS, pages 5998-6008, 2017.

[Velickovic et al., 2017] Petar Velickovic, Guillem Cucurul1, Arantxa Casanova, Adriana Romero, Pietro Lio, and Yoshua Bengio. Graph attention networks. arXiv: $M a-$ chine Learning, 2017.

[Vlahogianni et al., 2005] Eleni I Vlahogianni, Matthew G Karlaftis, and John C Golias. Optimized and metaoptimized neural networks for short-term traffic flow prediction: A genetic approach. Transportation Research Part C: Emerging Technologies, 13(3):211-234, 2005.

[Williams and Hoel, 2003] Billy M Williams and Lester A Hoel. Modeling and forecasting vehicular traffic flow as a seasonal arima process: Theoretical basis and empirical results. Journal of transportation engineering, 129(6):664-672, 2003.

[Wu and Tan, 2016] Yuankai Wu and Huachun Tan. Shortterm traffic flow forecasting with spatial-temporal correlation in a hybrid deep learning framework. arXiv preprint arXiv:1612.01022, 2016.

[Wu et al., 2019] Zonghan Wu, Shirui Pan, Guodong Long, Jing Jiang, and Chengqi Zhang. Graph wavenet for deep spatial-temporal graph modeling. IJCAI, 2019.

[Xingjian et al., 2015] SHI Xingjian, Zhourong Chen, Hao Wang, Dit-Yan Yeung, Wai-Kin Wong, and Wang-chun Woo. Convolutional lstm network: A machine learning approach for precipitation nowcasting. In NIPS, 2015.

[Yao et al., 2018] Huaxiu Yao, Xianfeng Tang, Hua Wei, Guanjie Zheng, Yanwei Yu, and Zhenhui Li. Modeling spatial-temporal dynamics for traffic prediction. arXiv preprint arXiv:1803.01254, 2018.

[Yu et al., 2018] Bing Yu, Haoteng Yin, and Zhanxing Zhu. Spatio-temporal graph convolutional networks: A deep learning framework for traffic forecasting. In IJCAI, 2018.

[Zhang et al., 2016] Junbo Zhang, Yu Zheng, Dekang Qi, Ruiyuan Li, and Xiuwen Yi. Dnn-based prediction model for spatio-temporal data. In ACM SIGSPATIAL, page 92, 2016.

[Zheng et al., 2019] Chuanpan Zheng, Xiaoliang Fan, Cheng Wang, and Jianzhong Qi. Gman: A graph multiattention network for traffic prediction. arXiv preprint arXiv:1911.08415, 2019. 